\title{
Estimation of Possible Profit/ Loss of a New Movie Using "Natural Grouping” of Movie Genres
}

\author{
Debaditya Barman \\ Assistant Professor, Department of Computer Science, University of GourBanga, Malda -732103, West Bengal, India \\ Email: debadityabarman@gmail.com \\ Dr. Nirmalya Chowdhury \\ Depart ment of Computer Science and Engineering, Jadavpur University, Kolkata - 700032, India \\ Email:nirmalya_chowdhury@yahoo.com
}

\begin{abstract}
Film industry is the most important component of entertainment industry. A large amount of money is invested in this high risk industry. Both profit and loss are very high for this business. Thus if the production houses have an option to know the probable profit/loss of a completed movie to be released then it will be very helpful for them to reduce the said risk. We know that artificial neural networks have been successfully used to solve various problems in numerous fields of application. For instance backpropagation neural networks have successfully been applied for Stock Market Prediction, Weather Prediction etc. In this work we have used a backpropagation network that is being trained using a subset of data points. These subsets are nothing but the "natural grouping" of data points, being extracted by an MST based clustering methods. The proposed method presented in this paper is experimentally found to produce good result for the real life data sets considered for experimentation.
\end{abstract}

Index Terms - Film industry; Film genre; Backpropagation network; ANN; MST Clustering; Natural Grouping.

\section{INTRODUCTION}

A movie [42], also called a film or motion picture, is a series of still or moving images. It is produced by recording photographic images with cameras, or by creating images using animation techniques or visual effects.

Films are cultural artifacts created by specific cultures, which reflect those cultures, and, in turn, affect them. It is considered to be an important art form, a source of popular entertainment and a powerful method for educating or indoctrinating citizens. The visual elements of cinema give motion pictures a universal power of communication.

The process of filmmaking has developed into an art form and has created an industry in itself. Film Industry is an important part of present-day mass media industry or entertainment industry (also informally known as show business or show biz). This industry [61] consists of the technological and commercial institutions of filmmaking: i.e. film production companies, film studios, cinematography, film production, screenwriting, preproduction, post production, film festivals, distribution; and actors, film directors and other film crew personnel.

The major business centers of film making are in the United States, India, Hong Kong and Nigeria. The average cost [43] of a world wide release of a Hollywood film or American film (including preproduction, film and post-production, but excluding distribution costs) is about $\$ 65$ million. It can be stretched up to $\$ 300$ million [44] (Pirates of the Caribbean: At World's End). World wide gross revenue [45] can be almost $\$ 2.8$ billion (Avatar). Profit-loss is found to vary from a profit [46] of $2975.63 \%$ (City Island) to a loss [47] of $1299.7 \%$ (Zyzzy x Road). So it will be very useful if we can develop a prediction system which can predict about Film's business potential.

Many artificial neural network based methods have been used to design for successful Stock Market Prediction [31], Weather Prediction [32] , Image Processing [33], and Time Series Prediction [1], and Temperature Prediction system [2] etc. In this work we have used Backpropagation neural network for prediction of profit/loss of a movie based on some predefined genres. Note that the performance of a backpropagation network mainly depends on choosing an appropriate set of data for training. Intuitively it can be said that the profit of a new movie will be similar to that of an old movie having similar values of genres and other parameters like overall rating giving by the viewers, reputation of the film distributors and present popularity of actor/actress performed for the film. With this idea in mind, we have found a natural grouping of the movies based on their genre values using an MST based clustering method. In this paper we have proposed a training method to train backpropagation neural network for prediction of possible business of a movie [36]. For training, we have chosen a subset of training data from entire training dataset based on the target movie's features. An MST based clustering method is used to group the movies. The formulation of the problem is presented in the next section. Sec. 2.1 
presents the term natural grouping in the context of clustering. Sec. 3 describes our proposed method. Experimental results on 25 movies selected randomly from a given database can be found in Sec. 4. Concluding remarks and scope for further work have been incorporated in section 5 .

\section{STATEMENT OF THE PROBLEM}

Every Film can be identified by certain film genres. In film theory, genre [48] refers to the method based on similarities in the narrative elements from which films are constructed. Most theories of film genre are borrowed from literary genre criticis $\mathrm{m}$. Some basic film genres are - action, adventure, animation, biography, comedy, crime, drama, family, fantasy, horror, mystery, romance, science-fiction, thriller, war etc. One film can belong to more than one genre. As an example the movie titled “Alice in Wonderland (2010)" belongs to [49] action, adventure, and fantasy genres.

Any film's success is highly dependent on its film genres. Other important factors are reputation of Film Studio or Production house and present popularity of casted actor/actress. We have considered these genres and the said factors as a Film's attributes. We can then collect the values for those attributes for several films released in the past. Based on these data we can estimate/ predict the possible future business of an upcoming Film.

We have used 20 movie genres like action, adventure, animation, biography, comedy, crime, documentary, drama, family, fantasy, history, horror, musical, mystery, romance, science fiction, sport, thriller, war, and western. Note that the factors such as the overall rating giving by the viewers, reputation of the film distributors and present popularity of actor/actress performed for the film, has been taken care of by the inclusion of the following 3 attributes- film distributor's reputation, overall rating and casting rating.

Our movie database consists of 395 Hollywood movies released in the year 2009, 2010 and 2011. We have chosen a subset of 25 movies from our movie database for testing purpose. Remaining 370 movies used for training of the backpropagation neural network. The movies that belong to this subset are then divided into a number of "natural groups" based on the similarity of the film's attributes as stated above. The notion of natural grouping is explained in the next subsection. Note that, one can expect desirable result if the neural network can be trained with those movies which have similar genre values as that of the target movie.

Note that Clustering can be used as a technique to find similarity or dissimilarity among the objects. Our objective here is to partition the training database into a number of "natural groups", such that, movies belonging to a particular group are similar to each other with respect to their attribute values and movies belonging to different groups are dissimilar on the same basis. Here our intention is to train the neural network with a data of a particular group of movies which are similar to that of the target movie.

\subsection{The importance of natural grouping in generation of training data sets}

Clustering in true sense is an unsupervised technique used in discovering inherent structure present in the set of objects. Clustering is a technique used to group data points of similar type from a heterogeneous collection of data points.

Let the set of patterns be $\mathrm{S}=\left\{\mathrm{d}_{1}, \mathrm{~d}_{2}, \mathrm{~d}_{3}, \ldots, \mathrm{d}_{\mathrm{m}}\right\} \in \mathrm{R}^{\mathrm{n}}$ where $d_{i}$ is the $i_{\text {th }}$ pattern vector corresponding to $i_{\text {th }}$ movie, $\mathrm{m}$ is the total number of movies in a given set of movies and $n$ is the dimensionality of the feature space. Since we have considered 23 movie attributes hence the value of $\mathrm{n}$ for our experiment is twenty three.

Let the number of clusters be $\mathrm{K}$. If the clusters are represented by $\mathrm{C}_{1}, \mathrm{C}_{2}, \mathrm{C}_{3}, \ldots, \mathrm{C}_{\mathrm{k}}$ then we assume:

P1. $C_{i} \neq$ f ori $=1,2, \ldots, K$

P2. $C_{i} \cap C_{j} \neq \Phi$, for $i \neq j$ and

P3. $U_{i=1}^{K} C_{i}=S$, where $\Phi$ represents null set.

Clustering techniques may broadly be divided into two categories: hierarchical and non-hierarchical. The non-hierarchical or partitional clustering problem deals with obtaining an optimal partition of $\mathrm{S}$ into $\mathrm{K}$ subsets such that some clustering criterion is satisfied. Among the non-hierarchical clustering techniques, the K-means (or C-means or basic Isodata) algorithm has been one of the more widely used algorithms. This algorithm is based on the optimization of a specified objective function. It attempts to minimize the sum of squared Euclidean distances between patterns and their cluster centers. It was shown in [19] that this algorithm may converge to a local minimum solution. Moreover it may not always detect the natural grouping in a given data set, though it is useful in many applications.

A lot of scientific effort has already been dedicated to cluster analysis problems, which attempts to extract the "natural grouping", present in a data set. The intuition behind the phrase "natural group" is explained below in the context of data set in $\mathrm{R}^{2}$.

For a data set $M=\left\{d_{1}, d_{2}, d_{3}, \ldots, d_{m}\right\} \in R^{2}$ obtain the scatter diagram of $\mathrm{M}$. By viewing the scatter diagram, what one perceives to be the groups present in $M$ is termed as natural groups of $\mathrm{M}$. For example, for the scatter diagram shown in Fig. 1.1(a), the groups that we perceive are shown in Fig. 1.1(b). Similarly for the scatter diagrams shown in Fig. 1.2 (a), the natural groups are as shown in Fig. 1.2(b).

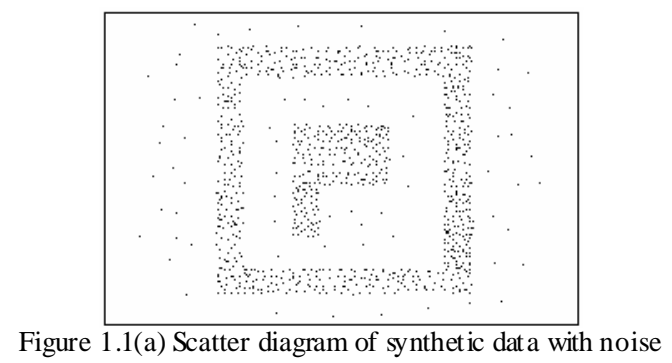




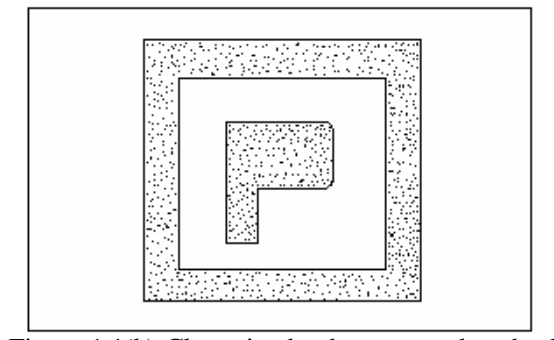

Figure 1.1(b) Clustering by the proposed method

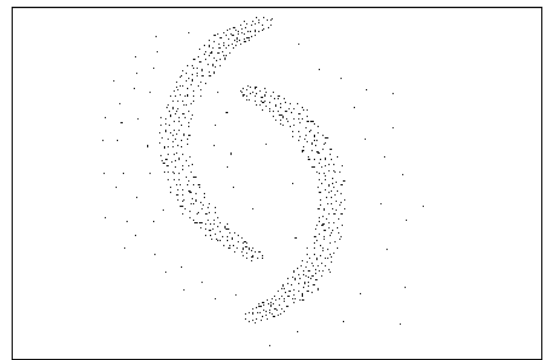

Figure 1.2(a) Scatter diagram of synthet ic data with noise

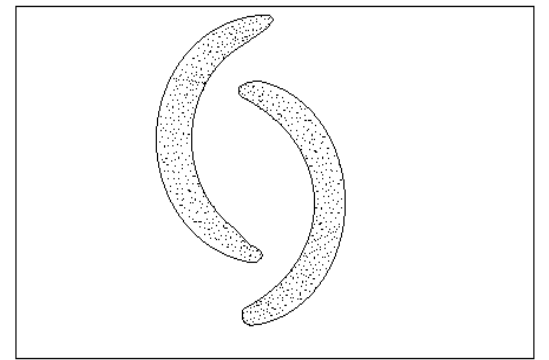

Figure 1.2 (b) Clust ering by the proposed method

Clustering techniques [18, 27-30] aim to extract such "natural groups" present in a given data set and each such group is termed as a cluster. So we shall use the term "cluster" or "group" interchangeably in this paper. Existing clustering techniques may not always find the natural grouping. The method for obtaining the natural groups in $R^{2}$ can also be extended to $R^{p}(p>2)$. Note that, the perception of natural groups in the data set is not possible for higher dimensional data. But the concept used for detecting the groups in $\mathrm{R}^{2}$ and $\mathrm{R}^{3}$ may also be applicable to higher dimensional data to obtain a mean ingful grouping.

In this paper we have proposed an algorithm that uses MST of data points for finding the "natural grouping” of the given set of movies to be used for training. The important characteristic of the proposed method is that, given a data set, it can obtain the partitions automatically without knowing the value of $\mathrm{K}$. It may be noted that a function which takes into account all the interpoint distances of $S$ is the sum of edge weights of minimal spanning tree of $S$, where the edge weight is taken to be the Euclidean distance. If we represent the sum of the edge weight of minimal spanning tree [21] of $\mathrm{S}$ by $\mathrm{l}_{\mathrm{m}}$ then it can be noted that $\mathrm{l}_{\mathrm{m}} \rightarrow \infty$ in probability as $\mathrm{m} \rightarrow \infty$ and $\frac{\mathrm{l}_{\mathrm{m}}}{\mathrm{m}} \rightarrow 0$ in probability as $\mathrm{m} \rightarrow \infty$.

Thus the threshold $\mathrm{T}$ for the cluster separation is taken to be equal to $T=\frac{l_{m}}{m}$, where $l_{m}$ is the sum of the edge weights (edge weight is taken to be the Euclidian distance) of minimal spanning tree of $\mathrm{S}$. Note that $\mathrm{T}$ is a function of interpoint distances in $S$ as well as the number of points $\mathrm{m}$.

Note that a similar such function is used in [38]. Our proposed method accepts movies as input and assigns then into different groups based on their attributes. MST of data points are used to compute the above mentioned threshold for cluster separation. The definition of MST and how it is used to obtain the natural grouping are described below.

Given a connected, undirected graph $\mathrm{G}=\langle\mathrm{V}$, E $>$, where $\mathrm{V}$ and $\mathrm{E}$ are set of vertices and edges respectively, the minimum spanning tree problem is to find a tree $\mathrm{A}=\left\langle\mathrm{V}, \mathrm{E}^{\prime}\right\rangle$ such that $\mathrm{E}^{\prime}$ subset of $\mathrm{E}$ and the cost of $\mathrm{A}$ is minimal. Note that a minimum spanning tree is not necessarily unique. It is important to remember that a tree over $|\mathrm{V}|$ vertices contains $|\mathrm{V}|-1$ edges. A tree can be represented by an array of this many edges.

In this method, at first the movies are considered as individual vertices. The weight of the edges connecting the vertices is nothing but the Euclidean distance between their respective pattern vectors.

Initially we consider an empty set A and at every stage the smallest edge not present in A should be selected. Since a tree does not have a cycle, a cycle cannot be formed while selecting the edges. The entire method is continued unless all the vertices (movies) are included in the MST. In this way a minimal spanning tree is formed from the given input movies as data points.

The next task is to find out the sum of the edge weights present in the spanning tree $\mathrm{A}$. Then the sum is divided by the no. of movies (given as input) to obtain the threshold $\mathrm{T}$ (as mentioned in the earlier section.).Then all edges whose weights are greater than $\mathrm{F} * \mathrm{~T}$, where $\mathrm{F}$ is a constant (In our experiment we choose $\mathrm{F}=1.175$, since this value provided consistently good results) are removed from the spanning tree $\mathrm{A}$, yielding few disjoint graphs. The vertices of the individual trees are categorized into same cluster. There may be trees with a single node that indicates that the node is a lone member in that particular cluster.

We have taken the Euclidean distances from our target movie to all the cluster centers (cluster's center can be obtained from the cluster obtained in the previous process). We have used to train our neural network with the movies belong to same cluster with minimum distance from our target movie. This trained neural network is used to predict business of the target movie.

Artificial neural network [24] learning methods provide a robust approach to approximating real-valued, discrete-valued, and vector-valued target functions. For certain types of problems, such as learning to interpret complex real-world sensor data, artificial neural networks are among the most effective learning methods currently known. Artificial neural networks have been applied in image recognition and classification [3], image processing [25], feature extraction from satellite images [4], cash forecasting for a bank branch [5], stock market prediction [22], decision making [6], temperature forecasting [7], atomic mass prediction [41], Prediction 
of Thrombo-embolic Stroke [8], time series prediction [9], forecasting groundwater level [10].

Backpropagation is a common method of teaching artificial neural networks about how to perform a given task. It is a supervised learning method. It is most useful for feed-forward networks [51].

Backpropagation neural network is successfully applied in image compression [11], satellite image classification [12], irregular shapes classification [13], email classification [14], time series prediction [34], bankruptcy prediction [15], and weather forecasting [35].

\section{THE PROPOSED METHOD}

As stated above we have used an MST based clustering method to find the "natural grouping" of a given set of movies that are used for training of the backpropagation network for prediction of possible profit / loss of a new movie. This method of grouping requires no apriory knowledge about the number of such natural groups. The intuition behind the phrase "natural groups" is explained in the context of data set in $\mathrm{R}^{2}$ in the Sec. 2.1.

It seems that if the backpropagation neural network is trained with those movies that are having similar attribute values to the target movie we may get better results than that of the normal training process. Possibly this type of training method will reduce the neural network's complexity, train ing time, computation time.

In this paper we have used the proposed training methods and also the normal training methods to train multilayer feed-forward neural network. We have compared the result obtained for the said two methods in Table 1 . In the normal training method (Experiment 1) we have used all the movies from training data set to train the neural network which is used to predict the profit percentage of a target movie. In our proposed training algorithms (Experiment 2) we have used MST of data points for finding the "natural grouping" of the given training set of movies. This MST of data points are used to compute the threshold for cluster separation. We have taken the Euclidean distances from our target movie to all the cluster centers (cluster's center can be obtained from the cluster obtained in the previous process). We have used to train our neural network with the movies belong to same cluster with minimum distance from our target movie. Note that, a multilayer feed-forward neural network consists of an input layer, one or more hidden layers, and an output layer.

Here the backpropagation algorithm [23] performs learning on the said multilayer feed-forward neural network. It iteratively learns a set of weights for prediction of the profit/ loss percentage (10 scales) label of instances. A typical multilayer feed-forward network is shown in Fig. 2.

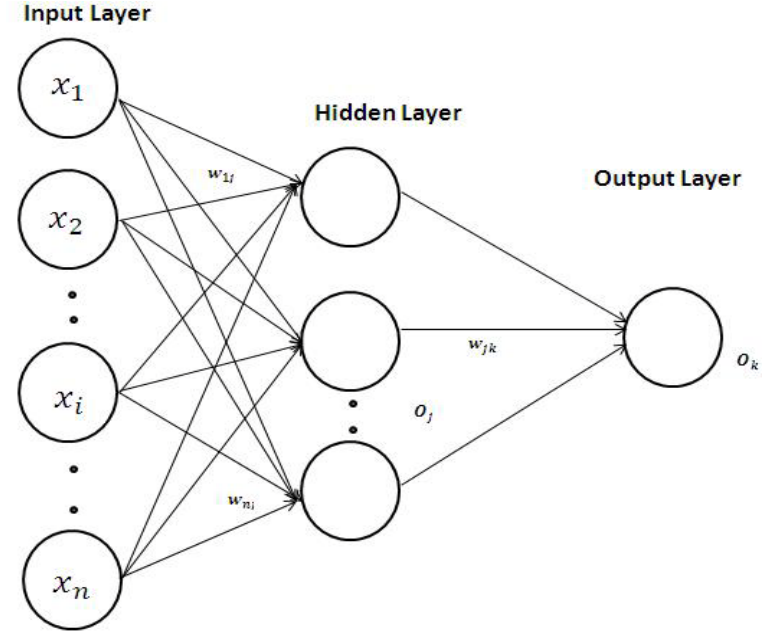

Figure. 2. A mult ilayer feed-forward neural net work.

Since we have 23 attributes (20 film genres, film distributor's reputation, overall rating and casting rating) to consider for this method, we need 23 nodes in the input layer. We have taken 10 nodes in the hidden layer and one node in the output layer. Note that there is no strict guide line to choose the number of nodes in a hidden layer. However, usually the number of nodes in the hidden layer is to be between the input layer size and the output layer size. We have experimented using different number of nodes in the hidden layer but we have obtained consistently good result in respect of learning speed with ten nodes in the hidden layer.

Note that input nodes have received real numbers that represents the values of the individual genres. A positive (negative) real number is generated at the output node which indicates the predicted profit (loss) of the movie of under consideration.

We have used 395 movie's data released from 2009 to 2011.For training of the said network we have used movies belong to the natural group which is nearest to our target movie. After the network has been successfully trained it can be used for prediction of profit/loss of new movie to be released. In our experiment we have taken some of the released movie (not taken in the training set) of 2010 for evaluating the efficiency of the trained backpropagation network. The experimental results are presented at the Sec. 4.

\subsection{Algorithm 1(To find natural grouping of the movies for training)}

The notations used in this algorithm are: G: graph containing all the vertices (all movies) and the edges (edge weights are the inter-vector distances), $\mathrm{W}_{\mathrm{ij}}$ : edge weights from vertex $i$ to vertex $j, T$ : threshold for the cluster separation (to be computed as stated above), $S_{i}, S_{j}$ : pattern vectors corresponding to the $\mathrm{i}_{\text {th }}$ and $\mathrm{j}_{\text {th }}$ movies respectively, $\Phi$ : null set. 
Step 1: $\boldsymbol{A} \leftarrow \boldsymbol{\Phi} / / A$ is a sub-graph of $G$

Step 2: $W_{i j}$ is calculated for all movies where $W_{i j}$ is the Euclidian distance from $S_{i}$ to $S_{j}$

Step 3: The edges of $G$ are sorted in increasing order of $W_{i j}$

Step 4: For any edge $E$ (considered in sorted order); if the endpoints of $E$ are disconnected in $A, E$ is added to $A$.

Step 5: Repeat step 4 till all the vertices (of $G$ ) are included in $A$.

Step 6: If $W_{i j}>1.175 * T$ then $W_{i j}=-1$ for all $i, j$

Step 7: For edges with $W_{i j} \neq-1$, the vertices $i, j$ connected by the edges are placed in the same cluster.

Step 8: For vertices where all the connecting edges have $W_{i j}=-1$ are placed in separate clusters where the said vertex (movie) is the sole member.

Step 9: Stop

Note that initially all the movies are considered to be in the same cluster. Then by using the threshold $\mathrm{T}$, the MST is splitted into small sub-graphs. Each of which corresponds to a separate cluster. The clusters may contain one or more than one members. These clusters are the natural groups obtained by the above mentioned MST based methods.

As stated above, there is 23 film attributes to form the pattern vector for each movie. Thus each movie is represented as a point in multidimensional feature space. Natural groupings of these data points then obtained by the proposed MST based methods as stated above.

3.2 Algorithm 2 (To select the appropriate group of movies for training)

The following algorithm selects the appropriate group of movies which will be used for neural network training of the backpropagation neural network.

Step 1: For a given target movie compute the Euclidian distance from its pattern vector to all the cluster centers obtained by the method stated in algorith $\mathrm{m}$.

Step 2: Find the cluster center for which the distance is minimum.

Step 3: Select the movies belonging to the cluster found in step 2 for train ing purpose.

Step 4: Stop

Algorithm 2, in fact, selects a subset of the movies given for training of the neural network. Here we have used backpropagation neural network for prediction of possible profit/loss of the movies. The backpropagation learning algorith m used in this work is stated below.

3.3 Algorithm 3 (For learning of the backpropagation neural network)

Input:

- $\quad D$, a data set consisting of the movies that belongs to the cluster $(C)$ which is nearest to our target movie.

- $\quad l$, the learning rate;

\section{Output:}

A trained neural network, which can predict profit percentage.

\section{Method:}

Step 1: In itialize all weights and biases in network;

Step 2: While terminating condition is not satisfied \{

Step 3: For each training instance $\boldsymbol{X}$ in $D\{/ /$ propagate the inputs forward:

Step 4: For each input layer unit $j$

Step 5: $O_{j}=I_{j} / /$ output of an input unit is its actual input value

Step 6: For each hidden or output layer unit $j\{$

Step 7: $I_{j}=\sum_{i} W_{i, j} O_{i}+\theta_{j} ; / /$ compute the net input of unit $j$ with respect to the Previous layer, $i$

Step 8: $\left.O_{j}=\frac{1}{1+e^{-I_{j}}} ;\right\} / /$ compute the output of each unit $j / /$ Back propagate the errors:

Step 9: For each unit $j$ in the output layer

Step 10: $E r r_{j}=O_{j}\left(1-O_{j}\right)\left(T_{j}-O_{j}\right)$; // compute the error

Step 11: For each unit $j$ in the hidden layers, fro m the last to the first hidden layer

Step 12: $E r r_{j}=O_{j}\left(1-O_{j}\right) \sum_{k} E r r_{k} w_{j k}$;// compute the error with respect to the next higher Layer, $k$

Step 13: For each weight $w_{i j}$ in network \{

Step 14: $\Delta w_{i j}=(l) E r r_{j} O_{i}$; // weight increment

Step15:. $\left.w_{i j}=w_{i j}+\Delta w_{i j}\right\}$; // weight update

Step 16: For each bias $\theta_{j}$ in network \{

Step 17: $\Delta \theta_{j}=(l) E r r_{j} ; / /$ bias increment

Step 18: $\theta_{j}=\theta_{j}+\Delta \theta_{j}$; $\} / /$ bias update

Step 19: \}\}

Step 20: Stop

At first, the weights in the network are initialized to small random numbers [23], bias associated with each unit also initialized to small random numbers.

The training instance from movie database is fed to the input layer. Next, the net input and output of each unit in the hidden and output layers are computed. A hidden layer or output layer unit is shown in Fig.3.

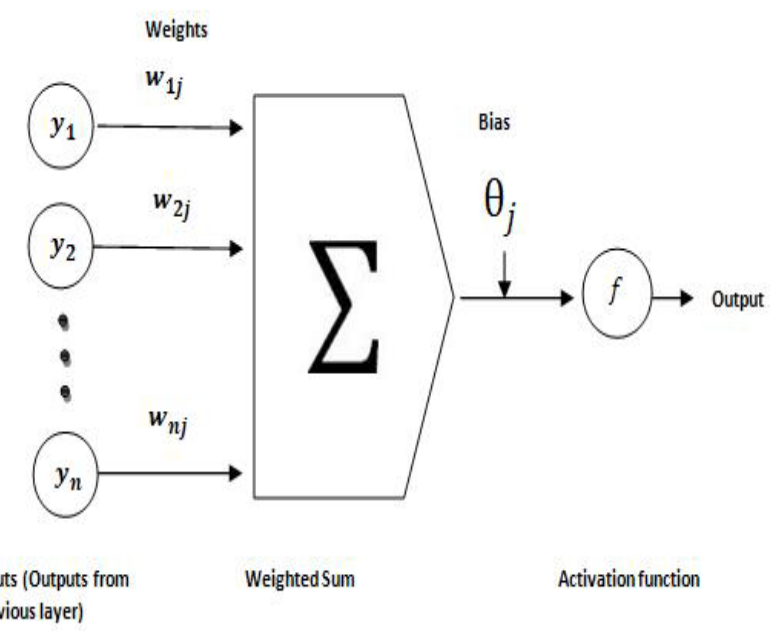

Figure 3. A hidden or output layer unit $j$ 
The net input to unit $\mathrm{j}$ is

$I_{j}=\sum_{i} w_{i j} O_{i}+\theta_{j}$

Where, $w_{i j}$ is the connection weight from unit $\mathrm{i}$, in the previous layer to unit $\mathrm{j}$; $\mathrm{O}_{\mathrm{i}}$ is the output of unit $\mathrm{i}$ from the previous layer; and $\theta_{j}$ is the bias of the unit.

As shown in the Fig. 2, each unit in the hidden and output layers takes its net input and then applies an activation function to it. The function (sigmoid) symbolizes the activation of the neuron represent by the unit. Given the net input $\mathrm{I}_{j}$ to unit $\mathrm{j}$, then $\mathrm{O}_{\mathrm{j}}$, the output of unit $\mathrm{j}$ co mputed as

$O_{j}=\frac{1}{1+e^{-I_{j}}}$

The error of each unit is computed and propagated backward. For a unit $\mathrm{j}$ in the output layer the error Err $_{\mathrm{j}}$ is computed by

$E r r_{j}=O_{j}\left(1-O_{j}\right)\left(T_{j}-O_{j}\right)$

where, $\mathrm{O}_{j}$ is the actual output of unit $j$, and $T_{j}$ is the known target value of the given training instance. The error of a hidden layer unit $\mathrm{j}$ is

$\operatorname{Err}_{j}=O_{j}\left(1-O_{j}\right) \sum_{k} \operatorname{Err}_{k} w_{j k}$

where, $w_{j k}$ is the weight of the connection from unit $j$ to a unit $k$ in the next higher layer, and $\operatorname{Err}_{k}$ is the error of unit $k$.

The weights and biases are updated to reflect the propagated errors. Weights are updated by the following equations, where $\Delta w_{i j}$ is the change in weight $w_{i j}$

$\Delta w_{i j}=(l) \operatorname{Err}_{j} O_{i}$

$w_{i j}=w_{i j}+\Delta w_{i j}$

$l$ is the learning rate. In our experiment it is 0.1 .

Biases are also updated, if $\Delta \theta_{\mathrm{j}}$ is the change in $\theta_{\mathrm{j}}$ then

$\Delta \theta_{j}=(l) E r r_{j}$

$\theta_{j}=\theta_{j}+\Delta \theta_{j}$

The weight and bias increments could be accumulated in variables, so that the weights and biases are updated after all of the instances in the training set have been presented. In our experiment we have used this strategy named epoch updating, where one iteration through the training set is an epoch.

The training stops when

- All $\Delta w_{i j}$ in the previous epoch were so small as to be below some specified threshold, or

- The percentage of instance misclassified in the previous epoch is below some threshold, Or

- A pre specified number of epochs have expired.

In our experiment we have specified the number of epochs as 1000 .

After successful training of the backpropagation neural network it is used for prediction of possible profit/ loss of a given movie selected form test data set of movies. The experimental results obtained are presented in the next section.

\section{EXPERIMENTAL RESULT}

We have carried out our experiments on a movie database containing 395 American films with 23 attributes (20 film genres, film distributor's reputation, overall rating and casting rating), released from 2009 to 2011. The data about investment and earnings of all the movies are obtained from Wikipedia (http://en.wikipedia.org) and rating and genres of the movies are obtained from imdb (http://www.imdb.com/).

We have used 370 movies out of the total 395 movies to train the neural network. The attributes of the remaining 25 movies are used as input of the trained network to predict the possible profit/ loss.

In our first experiment (Experiment 1), we have used all the 370 movies of the training data set to train the neural network. The attributes of the target movie from test set are used as input of the trained network to predict the possible profit/ loss.

Unlike Experiment 1, in our second experiment (Experiment 2), we have used an appropriate subset of the complete training set of movies to train the neural network for prediction of possible profit/ loss of each given movie selected from the test set. In fact for each movie for which the prediction of possible profit/ loss is sought, we compute an appropriate subset of movies from the training set by using our proposed MST based method to train the backpropagation neural network for prediction. Then the attributes of the target movie are used as input of the trained network to predict the possible profit/ loss. We have carried out our experiment with 25 movies from our test set.

The experimental results obtained from Experiment 1 and Experiment 2 are presented in Table1. In table 1, the first column presents name of the 25 movies from the test set selected for experimentation both with Experiment 1 and experiment 2. The second column shows the actual profits made by those movies. Percentages of possible profit/ loss predicted by the method used in experiment 1 are listed in Column 3 . Percentage of error committed by experiment 1 , for each movie has been narrated in column 4. Similarly column 5 and 6 represent prediction of possible profit/ loss and percentage of error respectively, obtained by our proposed method used in Experiment 2.

It can be shown from Table 1 that the possible profit/ loss prediction by Experiment 2 yield percentage error rate of less that $5 \%$ for 15 numbers of movies, $5-10 \%$ for 4 number of movies and greater than $10 \%$ for 6 numbers of movies. Note that for Experiment 1, predicted possible profit/ loss provided percentage error rate of less that $5 \%$ for 6 numbers of movies, $5-10 \%$ for 5 number of movies and greater than $10 \%$ for 14 numbers of movies 
TABLE 1. Experimental results (up to two decimal places)

\begin{tabular}{|c|c|c|c|c|c|}
\hline $\begin{array}{l}\text { Movie Name } \\
\text { (A) }\end{array}$ & $\begin{array}{l}\text { Actual } \\
\text { Profit in } \\
\text { percentage } \\
\text { (B) }\end{array}$ & $\begin{array}{l}\text { Profit percentage } \\
\text { ( Experiment 1) } \\
\text { (C) }\end{array}$ & $\begin{array}{l}\text { Percentage of } \\
\text { error } \\
\text { (Experiment 1) } \\
\text { (D) }\end{array}$ & $\begin{array}{l}\text { Profit percentage } \\
\text { ( Experiment 2) } \\
\text { (E) }\end{array}$ & $\begin{array}{l}\text { Percentage of error } \\
\text { ( Experiment 2) } \\
\text { (F) }\end{array}$ \\
\hline Blue Valentine & 1135.57 & 521.10 & 54.11 & 1178.65 & 3.79 \\
\hline Case 39 & 4.41 & 19.00 & 330.92 & 3.66 & 16.99 \\
\hline Conviction & -22.32 & -98.23 & 340.10 & -17.80 & 20.23 \\
\hline Cyrus & 41.77 & 48.41 & 15.90 & 44.31 & 6.08 \\
\hline $\begin{array}{l}\text { Clash of the } \\
\text { Titans }\end{array}$ & 294.57 & 263.59 & 10.52 & 308.06 & 4.58 \\
\hline Daybreakers & 157.08 & 190.17 & 21.06 & 169.28 & 7.76 \\
\hline $\begin{array}{l}\text { Dinner for } \\
\text { Schmucks }\end{array}$ & 25.23 & 81.74 & 224.01 & 25.08 & 0.60 \\
\hline Due Date & 225.72 & 290.06 & 28.51 & 230.20 & 1.99 \\
\hline Eat Pray Love & 240.99 & 212.72 & 11.73 & 215.43 & 10.61 \\
\hline Faster & 48.10 & 49.73 & 3.39 & 46.72 & 2.86 \\
\hline Grown Ups & 239.29 & 242.89 & 1.51 & 236.55 & 1.15 \\
\hline Hereafter & 163.00 & 164.80 & 1.11 & 170.98 & 4.90 \\
\hline Inception & 415.99 & 338.02 & 18.74 & 356.52 & 14.29 \\
\hline Leap Year & 71.62 & 65.63 & 8.37 & 80.63 & 12.58 \\
\hline $\begin{array}{l}\text { Life as We } \\
\text { Know It }\end{array}$ & 178.02 & 156.02 & 12.36 & 182.08 & 2.28 \\
\hline Megamind & 147.30 & 158.99 & 7.93 & 139.41 & 5.361 \\
\hline $\begin{array}{l}\text { My Soul to } \\
\text { Take }\end{array}$ & -16.10 & 21.85 & 35.78 & -10.51 & 34.68 \\
\hline Salt & 168.83 & 178.65 & 5.82 & 172.52 & 2.19 \\
\hline Tangled & 127.20 & 122.32 & 3.84 & 125.46 & 1.37 \\
\hline $\begin{array}{l}\text { The Bounty } \\
\text { Hunter }\end{array}$ & 240.81 & 269.06 & 11.73 & 235.17 & 2.34 \\
\hline $\begin{array}{l}\text { The } \\
\text { Expendables }\end{array}$ & 243.09 & 238.58 & 1.85 & 240.98 & 0.87 \\
\hline $\begin{array}{l}\text { The Kids Are } \\
\text { All Right }\end{array}$ & 767.65 & 805.31 & 4.91 & 751.27 & 2.13 \\
\hline $\begin{array}{l}\text { The Next Three } \\
\text { Days }\end{array}$ & 109.16 & 117.83 & 7.94 & 110.06 & 0.82 \\
\hline $\begin{array}{l}\text { The Twilight } \\
\text { Saga: Eclipse }\end{array}$ & 927.19 & 835.12 & 9.93 & 905.91 & 2.30 \\
\hline $\begin{array}{l}\text { Wall Street: } \\
\text { Money Never } \\
\text { Sleeps }\end{array}$ & 92.50 & 80.10 & 13.41 & 84.07 & 9.11 \\
\hline
\end{tabular}

It may also be noted that experiment 2 has provided a percentage error of less than $1 \%$ for two movies. Fig. 4 represents the results of experiment 1 and experiment 2 in the form of bar chart. Thus it may be concluded that our proposed method using appropriate subset of training data has provided much better result than that of the conventional method of using the complete training set. 


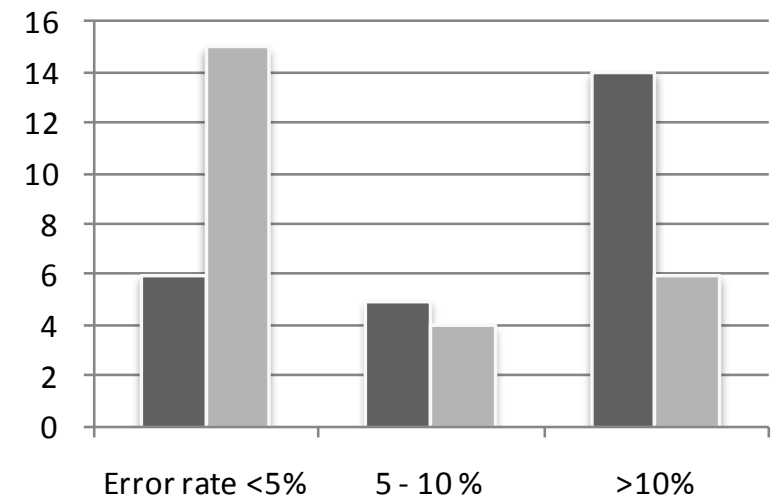

- Experiment 1 (Number of Movies)

Experiment 2 (Number of Movies)

Figure 4. Comparison between two experiment results

\section{CONCLUSION A ND SCOPE FOR FURTHER WORK}

It is obvious from the experimental result presented above that our proposed training method using a subset of movies (that are similar to the target movie in terms of film attributes) selected from the given training set has provided much better result in predicting the possible profit/ loss of a given target movie. Thus it may be concluded that it is better to use an appropriate subset of the training set rather than using complete training set (done for experiment 1) for better prediction of possible profit/ loss. It may be noted that the proposed method used in experiment 2 has provided an error rate of even less than $5 \%$ for 60 percent of the target movies selected for experimentation.

Note that, it is very difficult even for a human expert to predict the possible profit or loss of a new movie to be released. It seems that the genres of the movie play a significant role in the profit of the movie but it is very difficult to analytically establish the relation of the value of the genres of a given movie with the profit that it makes. In this paper we have attempted to develop a heuristic method using backpropagation neural network with an appropriate subset of the complete training set for training of the neural network to solve this problem.

Further research work can be conducted in the following areas. We can search for more genres and/or division of an existing genre into subgenres that may led to a higher success rate of prediction. Also we can conduct research for finding features of profitable movie trend in terms of some specific genres possibly using a psychological analysis of peoples' likings or interests in some specific kinds of movies.

\section{REFERENCES}

[1] R.J.Frank, N.Davey, S.P.Hunt , “Time Series Prediction and Neural Networks”, Journal of
Intelligent and Robotic Systems, Volume 31 Issue 1-3, May -July 2001, $91-103$.

[2] S. SanthoshBaboo and I.KadarShereef, "An Efficient Weather Forecasting System using Artificial Neural Network”, International Journal of Environmental Science and Development (IJESD), 2010, Vol.1(4): 321-326.

[3] C.C. Yang, S.O. Prasher, J.A. Landry, H.S. Ramaswamy and A. Ditommaso , "Application of artificial neural networks in image recognition and classification of crop and weeds", Canadian Agricultural Engineering, 2000, Vol. 42, No. 3, 147 $-152$.

[4] F. Farnood Ahmadia, M. J. ValadanZoeja, H. Ebadia, M. Mokhtarzadea, " The Application of Neural Networks, image Processing and CAD based environments facilities in automatic road extraction and vectorization from high resolution satellite images" The International Archives of the Photogrammetry, Remote Sensing and Spatial Information Sciences. Vol. XXXVII. Part B3b. Beijing 2008, 585 - 592.

[5] PremChand Kumar, EktaWalia " Cash Forecasting: An Application of Artificial Neural Networks in Finance ", International Journal of Computer Science \& Applications, 2006, Vol. III, No. I, 61 77.

[6] Hill, Tim, Leorey Marquez, Marcus O'Connor, and William Remus. "Artificial neural network models for forecasting and decision making." International Journal of Fo recasting 10, no. 1 (1994): 5-15.

[7] Hayati, Mohsen, and Zahra Mohebi. "Application of artificial neural networks for temperature forecasting." World Academy of Science, Engineering and Technology 28.2 (2007): 275-279.

[8] Shanthi, D., G. Sahoo, and N. Saravanan. "Designing an artificial neural network model for the prediction of thrombo-embolic stroke." International Journals of Biometric and Bioinformatics (IJBB) 3.1 (2009): 10-18.

[9] Frank, R. J., Neil Davey, and S. P. Hunt. "Time series prediction and neural networks." Journal of Intelligent and Robotic Systems 31.1-3 (2001): 91103.

[10] Sreekanth, P. D., N. Geethanjali, P. D. Sreedevi, Shakeel Ahmed, N. Ravi Kumar, and PD Kamala Jayanthi. "Forecasting groundwater level using artificial neural networks." Current science 96, no. 7 (2009): 933-939.

[11] Durai, S. Anna, and E. Anna Saro. "Image compression with back-propagation neural network using cumulative distribution function." World Academy of Science, Engineering and Technology 17 (2006): 60-64.

[12] Sapkal, A. T., C. Bokhare, and N. Z. Tarapore. "Satellite Image Classification using the Back Propagation Algorithm of Artificial Neural Network." Technical Article: 1-4.

[13] Shih-Wei Lin, Shuo-Yan Chou and Shih-Chieh Chen , " Irregular shapes classification by back- 
propagation neural networks ", The International Journal of Advanced Manufacturing Technology (2007), Volume 34, Issue 11-12, 1164-1172.

[14] TaiwoAyodele, Shikun Zhou, RinatKhusainov", Email Classification Using Back Propagation Technique ", International Journal of Intelligent Computing Research (IJICR), Volume 1, Issue 1/2, 2010, 3- 9.

[15] Odom, Marcus D., and Ramesh Sharda. "A neural network model for bankruptcy prediction."International Joint Conference on In Neural Net works, 1990, pp. 163-168. IEEE, 1990.

[16] TaiwoAyodele, Shikun Zhou, RinatKhusainov", Email Classification Using Back Propagation Technique ", International Journal of Intelligent Computing Research (IJICR), Volume 1, Issue 1/2, 2010, 3- 9.

[17] Sibson, Robin. "SLINK: an optimally efficient algorith $m$ for the single-lin k cluster method." The Computer Journal 16, no. 1 (1973): 30-34.

[18] Devijver, P.A. and J. Kittler., Pattern Recognition: A statistical Approach, Prentice-Hall International. HemelHemstead, Hertfordshire, UK, 1982.

[19] Selim, S.Z. and M.A. Is mail., K-means type algorithms: A generalized convergence theorem and characterization of local optimality. IEEE Trans. Pattern Anal. Mach. Intell. 6(1), 1984, 81-87.

[20] N. Chowdhury, C. A. Murthy: Minimal spanning tree based clustering technique: Relationship with Bayes Classifier. Pattern Recognition 30(11): 19191929 (1997)

[21] N. Chowdhury and C. A. Murthy, "Minimal Spanning Tree based clustering technique: relationship with Bayes Classifier”, Pattern Recognition, Vol. 30, No. 11, 1919-1929, 1997.

[22] Birgul Egeli, Asst. "Stock Market Prediction Using Artificial Neural Networks." Decision Support Systems 22: 171-185.

[23] Jiawei Han and MichelineKamber , “Data Mining: Concepts and Techniques”, 2nd edition, Page 328.

[24] Tom Mitchell, "Machine Learn ing ", page 81.

[25] Thai Hoang Le, "Applications of Artificial Neural Networks to Facial Image Processing ", InTechOpen, 2011, 213 - 240.

[26] Oded Z. Maimon, LiorRokach , "Data Mining And Knowledge Discovery Handbook”.

[27] Anderberg, M.R., "Cluster Analysis for Application”, Academic Press, Inc., NewYork, 1973.

[28] Jain, A.K. and R.C. Dubes., Algorithms for Clustering Data. Prentice-Hall, Englewood Cliffs, NJ, 1988.

[29] Spath, H., Cluster Analysis Algorithms. Ellis Horwood, Chichester. UK, 1980.

[30] Tou, T.J. and C.R. Gonzalez., Pattern Recognition Principles. Addison-Wesley, Reading, MA, 1974.

[31] Bernd Freisleben "Stock Market Prediction with Backpropagation Networks" Proceedings of the 5th international conference on Industrial and engineering applications of artificial intelligence and expert systems, 1992, 451-460.

[32] Gill, J.; Singh, B.; Singh, S.;"Training back propagation neural networks with genetic algorith m for weather forecasting" 8th International Symposium on Intelligent Systems and Informatics (SISY), 2010, 465 - 469.

[33] McClellan, G.E.; DeWitt, R.N.; Hemmer, T.H.; Matheson, L.N.; Moe, G.O.; "Multispectral imageprocessing with a three-layer backpropagation network". International Joint Conference on Neural Networks, 1989. IJCNN.vol.1, 151 - 153.

[34] Frank M. Thiesing, Ulrich Middelberg and Oliver Vornberger,"Parallel back-propagation for the prediction of time series ", 1 st European PVM Users Group Meeting, Rome, October 9-11, 1994.

[35] S. SanthoshBaboo and I.KadarShereef, "An Efficient Weather Forecasting System using Artificial Neural Network ", IJESD 2010 Vol.1 (4): 321-326.

[36] D. Barman, Dr. N. Chowdhury, “An ANN Based Movie Business Prediction Method using a Subset of Training Data”, Proceedings of the conference "International Conference on Computer Applications 2012” 2012 Volume 02, 166-171.

[37] D. Barman, Dr. N. Chowdhury, "A Method of Movie Business Prediction using Artificial Neural Network”, ICSEA-2011)”, 54-59.

[38] D. Chaudhuri, C. A. Murthy and B.B. Chaudhuri, "Finding a Subset of Representative Points in a Data set”, IEEE SMC, 24 (9), 1994, 1416-1424.

[39] N. Chowdhury, Premananda Jana: Finding the Natural Groupings in a Data Set Using Genetic Algorithms. AACC 2004: 26-33.

[40] P. Jana, N. Chowdhury: Finding the Natural Grouping in a Data Set Using MST of the Data Set. IICAI 2005: 2056-2071.

[41] "Atomic Mass Prediction with Articial Neural Networks" by xuru.org.

[42] http://en.wikipedia.org/wiki/Film.

[43] http://www.the-numbers.com/glossary.php.

[44] http://en.wikipedia.org/wiki/List_of_most_expensi ve_films.

[45] http://en.wikipedia.org/wiki/List_of_highestgrossing_films.

[46] http://en.wikipedia.org/wiki/City_Island_\%28film $\% 29$.

[47] http://en.wikipedia.org/wiki/Zyzzy x_Road.

[48] http://en.wikipedia.org/wiki/Film_genre.

[49] http://www.imdb.com/title/tt0499549/.

[50] http://en.wikipedia.org/wiki/List_of_American_fil ms_of_2010.

[51] http://en.wikipedia.org/wiki/Backpropagation.

[52] http://www.film-releases.com/film-releaseschedule-2010.php.

[53] http://www.imdb.com/title/tt1126591/.

[54] http://www.imdb.com/title/tt1433108/.

[55] http://www.imdb.com/title/tt0758752/.

[56] http://en.wikipedia.org/wiki/Tron:_Legacy. 


\section{Authors' Profiles}

Debaditya Barman: Deabaditya Barman has obtained his B.E. (2008) and M.E. (2012) in Computer Sc. and Engineering from Jadavpur University, India. After M.E., he has completed EPBABI (Executive Programme in Business Analytics and Business Intelligence) from Indian Institute of Management, Ranchi, India. At present, he is working as Assistant Professor in the department of Computer Science, University of Gour Banga, India. His main areas of research interest are Machine Learning, Business Analytics, Business intelligence, Data mining and Opin ion mining.

NirmalyaChowdhury: Nirmalya Chowdhury did his Ph.D. in Engineering from Jadavpur University, India in 1997. At present, he is working as Associate Professor in the department of Computer Science and Engineering, Jadavpur University, India. His fields of research include Pattern Recognition, Soft Computing, Natural Language Processing and Bioinformatics. 